\title{
Development, roll-out and impact of Xpert MTB/RIF for tuberculosis: what lessons have we learnt and how can we do better?
}

\author{
Heidi Albert ${ }^{1}$, Ruvandhi R. Nathavitharana $\mathbb{1}^{2}$, Chris Isaacs ${ }^{3}$, Madhukar Pai ${ }^{4}$, \\ Claudia M. Denkinger ${ }^{2,3}$ and Catharina C. Boehme ${ }^{3}$
}

Affiliations: ${ }^{1}$ FIND, Cape Town, South Africa. ${ }^{2}$ Division of Infectious Diseases, Beth Israel Deaconess Medical Center, Harvard Medical School, Boston, MA, USA. ${ }^{3}$ FIND, Geneva, Switzerland. ${ }^{4}$ Dept of Epidemiology, Biostatistics and Occupational Health, McGill University, Montreal, QC, Canada.

Correspondence: Heidi Albert, FIND, Waverley Business Park, 17-107 Wyecroft Road, Mowbray, Cape Town, 7925, South Africa. E-mail: heidi.albertafinddx.org

ABSTRACT The global roll-out of Xpert MTB/RIF (Cepheid Inc., Sunnyvale, CA, USA) has changed the diagnostic landscape of tuberculosis (TB). More than 16 million tests have been performed in 122 countries since 2011, and detection of multidrug-resistant TB has increased three- to eight-fold compared to conventional testing. The roll-out has galvanised stakeholders, from donors to civil society, and paved the way for universal drug susceptibility testing. It has attracted new product developers to TB, resulting in a robust molecular diagnostics pipeline. However, the roll-out has also highlighted gaps that have constrained scale-up and limited impact on patient outcomes. The roll-out has been hampered by high costs for underfunded programmes, unavailability of a complete solution package (notably comprehensive training, quality assurance, implementation plans, inadequate service and maintenance support) and lack of impact assessment. Insufficient focus has been afforded to effective linkage to care of diagnosed patients, and clinical impact has been blunted by weak health systems. In many countries the private sector plays a dominant role in TB control, yet this sector has limited access to subsidised pricing. In light of these lessons, we advocate for a comprehensive diagnostics implementation approach, including increased engagement of in-country stakeholders for product launch and roll-out, broader systems strengthening in preparation for new technologies, as well as quality impact data from programmatic settings.

@ERSpublications

A comprehensive diagnostic solution approach including systems strengthening is essential for TB diagnostics impact http://ow.ly/uWSy300CfJT

Received: March 152016 | Accepted after revision: May 142016 | First published online: July 132016

Conflict of interest: Disclosures can be found alongside the online version of this article at erj.ersjournals.com

Copyright OERS 2016. ERJ Open articles are open access and distributed under the terms of the Creative Commons Attribution Non-Commercial Licence 4.0. 


\section{Introduction}

Recent innovations in "near patient" testing technologies for tuberculosis (TB) and HIV have brought game-changing potential to the care and treatment of the two epidemics. However, the roll-out of new diagnostic technologies, such as Xpert MTB/RIF (Cepheid Inc., Sunnyvale, CA, USA), can highlight critical gaps that may limit scale-up and patient impact. However, these can only be fully realised when symptomatic individuals are appropriately screened and referred for testing with new technologies and diagnosed patients are linked successfully to appropriate care and treatment. We reflect upon the roll-out of Xpert MTB/RIF as a case study to highlight essential elements necessary to achieve maximal impact, and put forward a set of requirements that should be met for roll-out of future diagnostic technologies to ensure maximal impact.

\section{Search strategy and selection criteria}

References for this review were identified through searches of PubMed for articles published from January 2010 to May 2015 by use of the terms: "Xpert MTB/RIF", “GeneXpert", "Xpert", "tuberculosis", "diagnostics" and "implementation". Relevant articles, reports and guidelines published between 2007 and 2015 were identified through searches in the authors' personal files. Articles resulting from these searches and relevant references cited in those articles were reviewed. Articles published in English were included.

\section{Product development}

In 2006, FIND (Geneva, Switzerland), a product development partnership, identified the GeneXpert platform as the most promising technology for a next generation TB test, and executed a co-development agreement with Cepheid and the University of Medicine and Dentistry of New Jersey (Newark, NJ, USA) to develop a TB test based on the automated GeneXpert platform, supported by funding from the Bill and Melinda Gates Foundation (Seattle, WA, USA) and the National Institute of Allergy and Infectious Diseases (Rockville, MD, USA). A bold decision was made to combine TB case detection with drug susceptibility testing, and a single cartridge was developed to detect Mycobacterium tuberculosis, as well as rifampin resistance. In April 2009, the product received initial regulatory approval (European CE-IVD) and evaluation and demonstration trials began the following month. As part of the agreement with Cepheid, FIND leveraged donor funding to negotiate a pricing model aimed at a $75 \%$ cost reduction for the public health sector in low- and middle-income countries.

\section{Policy and regulatory guidance}

As shown in figure 1, following Xpert MTB/RIF evaluation in 2009 [1] and field demonstration in 2010 [2], a World Health Organization (WHO) expert group meeting and global consultation were held in late 2010.

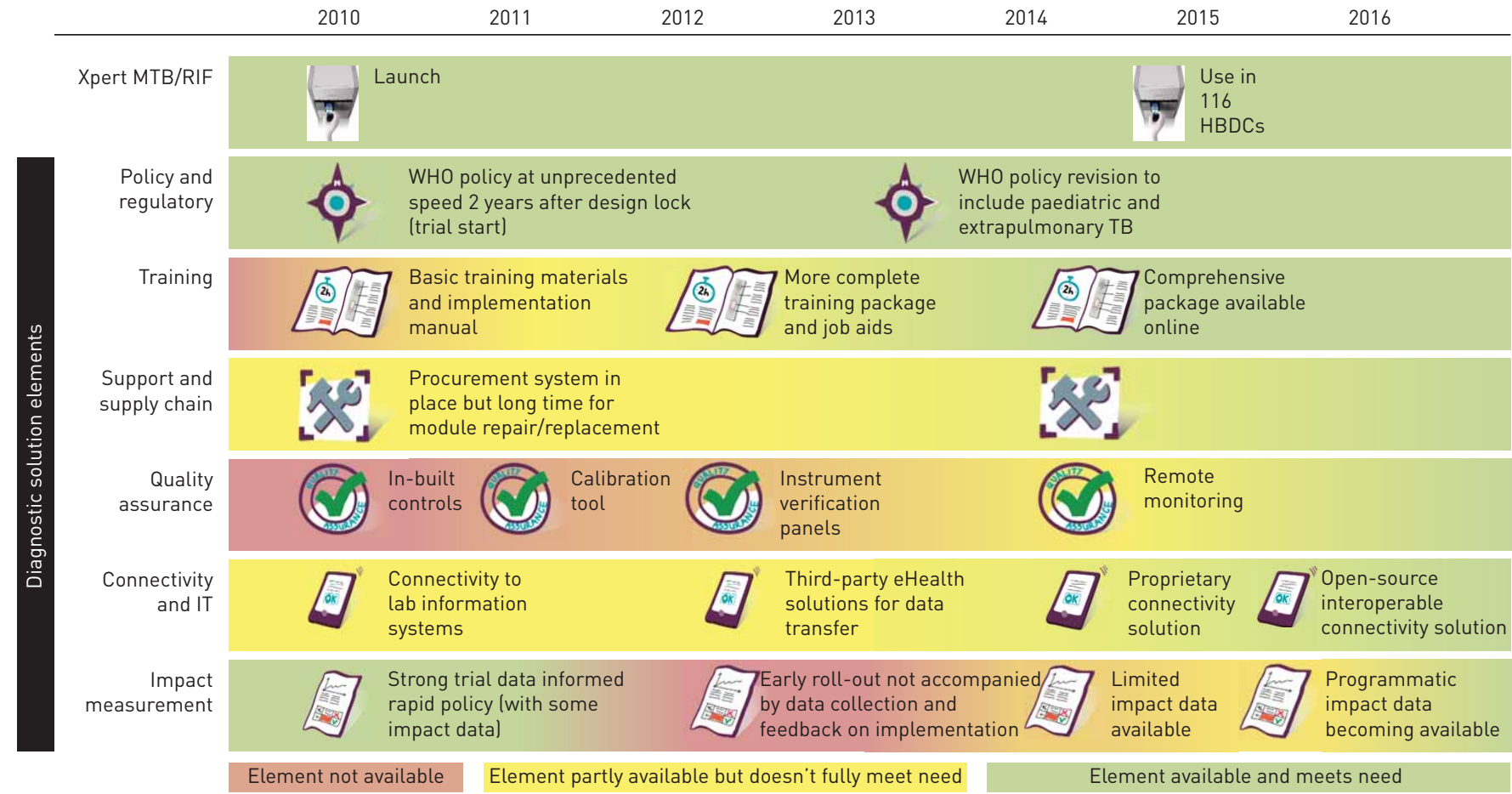

FIGURE 1 Timeline of availability of required elements for Xpert MTB/RIF implementation. HBDCs: high-burden developing countries; WHO: World Health Organization; TB: tuberculosis. 
This efficient global policy process was based on extensive multi-country study data from various epidemiological settings, and translated quickly into national policy [3]. In 2010, WHO endorsed the Xpert MTB/RIF product and recommended that Xpert: 1) should be used as the initial diagnostic test in individuals suspected of multidrug-resistant (MDR)-TB or HIV-associated TB (strong recommendation); and 2) may be used as a follow-on test to microscopy in settings where MDR and/or HIV is of lesser concern, especially in smear-negative specimens (conditional recommendation, recognising major resource implications) [4].

Translation of global policy into actionable implementation plans at the country level, however, proved slower and impact data was not generated. RAmSAY et al. [5] concluded that the importance of advocacy post-WHO endorsement of new TB diagnostics was underestimated, and suggested that a specific country and donor engagement plan was required to ensure translation of global policy into actionable implementation plans. In 2013, a WHO policy revision for Xpert was published [6] with an accompanying revised implementation manual [7]. The 2013 policy revision provided expanded and updated policy recommendations that may have influenced expanded use of Xpert MTB/RIF (including recommendations for use in paediatric and extrapulmonary TB). The updated manual reiterated the need for countries to conduct an impact assessment, but did not provide detailed guidance in this regard.

\section{Pricing and uptake}

When Xpert MTB/RIF was launched in 2010, the initial negotiated price was US\$17000 for a four-module GeneXpert instrument (with desktop computer) and US\$16.86 per Xpert MTB/RIF cartridge. The launch price was tied to a minimum manufacturing volume and was negotiated by FIND for use in the public sector and non-governmental organisations (NGOs) in 145 high-burden developing countries (HBDCs). As shown in figure 1, initial uptake was slow and cost was a major barrier to scale-up.

In August 2012, PEPFAR (President's Emergency Plan for AIDs Relief), USAID (United States for International Development), UNITAID, and the Bill and Melinda Gates Foundation finalised an agreement with Cepheid to further reduce the negotiated price of the Xpert MTB/RIF cartridge for eligible customers on the FIND country list from US\$16.68 to US\$9.98, direct cost from the manufacturer and pre-paid goods. Around the same time, the South African government took the decision to scale-up the test across the country. Through these developments, the trajectory of Xpert uptake shifted substantially (figure 2). From 2010 to the end of 2015, a total of 21549 instrument modules and more than 16.2 million Xpert MTB/RIF cartridges have been procured by eligible customers in the public sector and NGOs, covering 122 HBDCs (personal communication; Cepheid Inc.). Funding for scale up of Xpert MTB/RIF has relied heavily on donor funding in the majority of HBDCs [7]. The South African

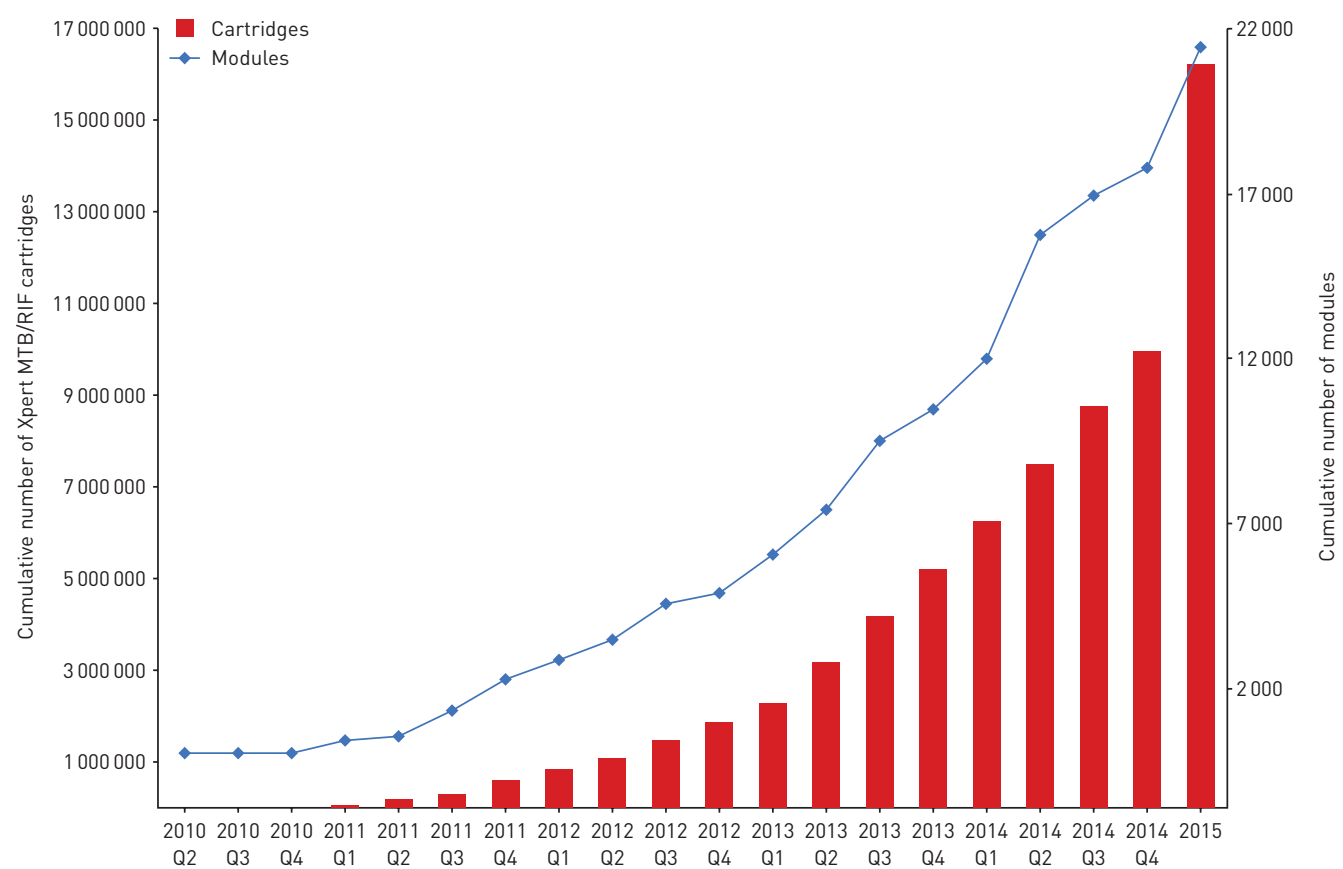

FIGURE 2 Procurement of Xpert MTB/RIF modules and cartridges under concessionary pricing by quarter (Q) in 2010-2015 (Cepheid data). 
programme is now primarily funded by the government, whereas most countries remain reliant on external donor funding, causing concern for long-term sustainability and future implementation of other technologies.

By 2014, 19 (86\%) out of 22 HBDCs reported an Xpert MTB/RIF implementation plan [8]. Data from a recent survey of 24 countries provides some indication of the completeness of these plans, and the extent to which they have been translated into practice [9]. Of the 24 countries, 14 recommend use of rapid molecular testing as the initial diagnostic test for people at risk of MDR-TB or HIV-associated TB, and eight countries have national policies with rapid molecular tests as a replacement for smear microscopy as the initial diagnostic test for presumptive TB in adults and children (figure 3) [8]. Only five countries have recommended the use of Xpert MTB/RIF for the diagnosis of paediatric TB, and four for extra-pulmonary TB. Quantifying the extent to which implementation of diagnostic policies has been achieved proved difficult; nonetheless, the authors conclude that significant gaps exist, and that broader coverage of Xpert MTB/RIF and other new rapid tests are needed in the majority of countries surveyed.

\section{Training}

Although well-controlled studies suggest that 1-2 days of training is adequate to establish proficiency on GeneXpert, field experience has shown that more comprehensive training, combined with regular monitoring and on-site supervision, has been necessary to improve: the quality of the test and software use; instrument maintenance; troubleshooting; the ability to follow guidelines; and communication of results for linkage to care. Training is particularly critical during the first 2 months of use (personal communication; V. Harris, FIND, Geneva, Switzerland).

Although the manufacturer's training materials were available at the time of product launch, several implementing partners developed custom materials to complement those provided by the manufacturer. These materials address issues of quality assurance, recording and reporting results, and specimen collection; many were not developed until 2-3 years after product launch. To standardise the content of the various training materials available, the Global Laboratory Initiative published a modular training package for Xpert MTB/RIF in early 2014. In future, a comprehensive training package, with associated tools, should be available in key languages at product launch to enable high-quality training to be delivered rapidly in most countries. Improvements in maintenance, troubleshooting errors and adherence to the recommended national algorithm were shown following 5 days of comprehensive training based on the Global Laboratory Initiative training package, combined with advanced technical support to sites (personal communication; V. Harris). Independent of the training materials for instrument operators, the largely absent focus on provider training has limited demand for testing, and has been now added to the Global Laboratory Initiative training package.

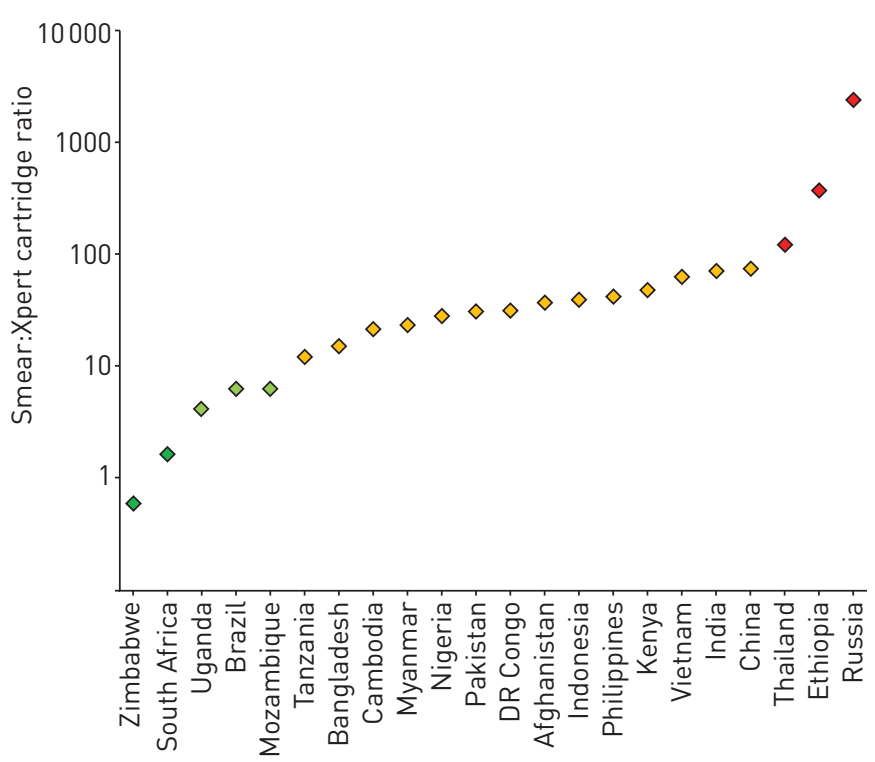

FIGURE 3 Uptake of Xpert MTB/RIF in 22 high-burden countries, comparison of Xpert cartridge procurement with smear volumes. Data from [8]. 


\section{Quality assurance}

In reference or referral laboratories, the essential elements of quality assurance are well accepted and clearly articulated in international standards; however, there has been much debate as to whether these stringent requirements are necessary or even feasible for peripheral laboratories (and non-laboratory testing sites) that are implementing Xpert MTB/RIF. Global Laboratory Initiative guidance on requirements for quality assurance for the Xpert MTB/RIF assay have been published, and include information on routine maintenance activities, new lot quality control testing, instrument verification, quality indicator monitoring and external quality assurance, including on-site supervision and proficiency testing. This guidance only became available 3 years after product launch, and implementation of several of these recommended elements remains challenging.

Several proficiency testing panels for Xpert MTB/RIF have been evaluated [10], but the possibility to enrol in external proficiency testing programmes only became available in 2013. A pilot proficiency testing programme was initiated by the US Centers for Disease Control and Prevention (Atlanta, GA, USA) in 2013 using dried culture specimens, and currently includes 193 testing sites in 11 countries, with a total of 810 panels shipped to date (personal communication; K. De Gruy, Centers for Disease Control and Prevention, Atlanta, GA, USA). South Africa's National Health Laboratory Service runs an external quality assurance programme using web-based reporting. INSTAND (Düsseldorf, Germany) also provides an Xpert MTB/RIF external quality assurance programme. Procurement of external proficiency testing panels is prohibitively expensive for many countries. Alternatively, panels may be locally manufactured at reference laboratories and distributed to testing sites, although standardised protocols are not yet published to guide countries.

On-site supervision is essential to maintain proficiency and check that regular maintenance activities are being consistently performed, yet they are performed irregularly in many countries. The availability of appropriately qualified staff with transport to sites, data management and linking non-conformities to corrective actions are major challenges, especially given the relatively large number and decentralised location of Xpert sites in many countries.

Routine monitoring of quality indicators, such as $M$. tuberculosis positivity rate, rifampicin resistance rate, error rate and turnaround time, is perhaps the most essential quality assurance activity, since it directly reflects routine testing. Routine quality monitoring can enable targeted supervision to poorly performing sites, allow early troubleshooting or servicing interventions, and contribute to good stock management. The inability to easily monitor quality indicators directly using the GeneXpert instrument software, and rapid pace of scale-up of instrument installations in some countries, have hampered attempts to monitor quality indicators. This has led to connectivity solutions based on the development of open-source remote monitoring systems, as described later.

\section{Infrastructure}

The original GeneXpert technology was targeted to the district or sub-district level in facilities with stable power, temperature control and protection from dust, heat and humidity. The challenges of operation in these settings became apparent as the technology rolled out in HBDCs. Stable power supply is a challenge in many settings [11], necessitating solutions that range from installation of uninterruptible power sources to serially connected car batteries, inverters and solar power systems. Countries have reported difficulty in local procurement of appropriate power systems. Furthermore, servicing and repair of a system purchased outside the country of intended use may be difficult. Environmental issues such as dust also resulted in high rates of module failure in some settings [12].

These challenges have meant that although the Xpert MTB/RIF test has enabled more decentralised drug susceptibility testing than ever before, in most HBDCs the technology is not implementable at the level of the microscopy centre, where most TB management services are focused. Responding to these realities, in July 2015 Cepheid and FIND unveiled a new generation instrument called GeneXpert Omni; described as the world's first portable, point-of-care, molecular diagnostic platform that is battery-operated, wireless and web-enabled.

\section{Maintenance and technical support}

Equipment maintenance and servicing is a notorious challenge in many low- and middle-income countriess. Higher than expected rates of module failure have been reported for Xpert in a number of settings. CRESWELL et al. [13] reported unsuccessful test rates of 10.6\% (range 5.9-16.3\%) in nine countries implementing Xpert MTB/RIF. RAIZADA et al. [12] reported initial test failure rates in India of 7.2\% (range 4-17\%), and high levels of module failure (11.36\%) were reported from South Africa with a median time to module failure of 452 days [14]. Spare modules were not available in the majority of countries, leading to extended downtimes (>3 months in $20 \%$ of reported cases) and additional local expenses, including complex processes for customs clearance. Although extended warranties are now available, a recent survey 
of implementers revealed low utilisation of extended warranties, with lack of funding as the most common reason (personal communication; H. Albert, FIND, Geneva, Switzerland). There is a need to strengthen the networks of authorised service providers to ensure adequate coverage and quality of diagnostic services. Due to the negotiated public sector pricing, normal market dynamics have not been applied to Xpert MTB/ RIF, with low margins for authorised service providers and centralised decision-making around test procurement. The current margins to service providers may be inadequate to meet basic needs in some settings, while customers may be unable to pay additional service costs.

Availability of replacement modules and spare parts in countries is increasingly being provided by Cepheid and could help to alleviate this problem. Nonetheless, the peripheral location and increasing numbers of instruments point to instrument service and maintenance as major future challenges, especially when next generation technologies become available that enable even greater decentralisation. Provision of additional services, such as web tracking of service and maintenance status of instruments, support for shipping and distribution of supplies, and support to install and maintain connectivity solutions, have been identified as key demands by users; innovative solutions will be needed to meet these demands.

\section{Supply chain}

Global stocks of Xpert MTB/RIF cartridges ran out in 2012 and 2013 due to inadequate production capacity; this seriously hindered roll-out. Local and national level stocks have run out frequently as a result of supply chain systems' weaknesses in many countries. In addition, an overestimation of cartridge needs, lack of coordination among various donors and complex distribution channels are commonly experienced. The requirement for cool storage of cartridges $\left(2-30^{\circ} \mathrm{C}\right)$ means that more frequent distribution from a central storage facility is needed, leading to increased cost and complexity. Supply forecasts based on actual consumption are not done universally, and overestimation of cartridge needs (by external partners and donors as well as by Ministry of Healths) remains a problem. While global procurement data of instruments and cartridges suggests reasonable usage of available instrument capacity, with an average of 611 cartridges sold per instrument module in 2014, or approximately three tests per working day (personal communication; Cepheid Inc.), these data are likely to overestimate average routine consumption and country experience suggests substantial under-utilisation of instrument capacity, particularly during early implementation [13].

Effective supply chain management remains an intractable problem, and may result in interruption in service delivery and wastage of resources. While remote monitoring may assist, attention needs to be paid to building local capacity to use available data to inform procurement and distribution decisions.

\section{Connectivity and IT}

Many of the implementation challenges described above may be ameliorated by the introduction of connected diagnostics and so-called eHealth solutions. eHealth solutions can serve as a catalyst to overcome specific constraints and can help increase the impact of a known intervention. However, to date, experience with implementation of eHealth solutions has been fragmented and variable, with a lack of data demonstrating impact on healthcare delivery and patient outcomes $[15,16]$. Connectivity within diagnostic platforms has become an evident pre-requisite [17].

At the time of introduction of Xpert, the only option for connectivity was to connect instruments to Laboratory Information Management Systems through an ethernet port on the device PC. However, since GeneXpert in Laboratory Information Management Systems was mostly being rolled out in peripheral sites without hard-wired systems, many sites continued to use slow, error prone, paper-based recording and communication of results, with South Africa a notable exception. As a consequence, valuable generated data were never used to inform patient care or surveillance efforts and much of the operational and quality data remain under-utilised and possibly lost [18].

Due to demands from countries to have greater access to GeneXpert data (for linkage to patient care, remote monitoring of instrument use and performance at sites, and to inform supply chain management), independent, open-source mobile health tools, including stand-alone connectivity hardware and software, were developed specifically for Xpert MTB/RIF. These tools were designed to meet the needs of low-resourced countries, where internet is often unavailable or unreliable, and Laboratory Information Management Systems or Electronic Medical Records are not widely used.

Several tools were developed by groups from Interactive Research and Development (XpertSMS, Karachi, Pakistan), Université Catholique de Louvain (GenXchange, Louvain-la-Neuve, Belgium) and Abt Associates (GX Alert, Cambridge, MA, USA). XpertSMS has been rolled out in TB REACH projects in a number of countries, including Pakistan and Bangladesh, GenXchange has been rolled out at more than 50 sites across the Democratic Republic of Congo and Cameroon, and GX Alert has been implemented in 23 countries (from small pilot projects to large-scale roll-outs), and is reportedly installed in $<10 \%$ of GeneXpert 
instruments in HBDCs. To gain visibility of data for technical support, quality assurance and supply chain purposes, Cepheid developed its own remote connectivity solution (Cepheid RemoteXpert), which was launched in early 2015 (and later renamed Cepheid Cloud Control). However, the initial version of this system has excluded patient identifying data, meaning that it would not support reporting of patient results.

Global initiatives by WHO and partners are underway to develop and implement standards for diagnostic connectivity and promote interoperability of solutions [18, 19]. Furthermore, connectivity solutions are needed for the entire range of laboratory tests in the diagnostic cascade. The list includes new rapid diagnostic tests as well as more conventional approaches, such as culture, drug susceptibility testing and line-probe assays [20]. Interconnecting these diagnostic devices, enabling the consolidated reporting of diagnostic test results and integrating this information with clinical indicators are key challenges. The Connected Diagnostics Initiative, initiated by FIND and the Innovative Support to Emergencies Diseases and Disasters (Sunnyvale, CA, USA), is accelerating this process by providing technical toolkits and assistance for diagnostic manufacturers, as well as developing open source platforms which are needed for data collection, access and linkage to care. The Connected Diagnostics Initiative platform will be launched in early 2016, allowing centralised aggregation of data from diagnostics regardless of manufacturer.

Successful implementation of such toolkits in a holistic way will involve a lengthy process of change management at many levels, requiring commitment, a roadmap and pro-active champions.

\section{Gaps in the TB care cascade}

Without efficient processes in place to screen and refer presumptive TB patients for testing, and ensure linkage of diagnosed patients to appropriate care and treatment (i.e. a test and treat strategy), the impact of improved diagnosis will remain unrealised. Diagnostic delays are common and may be related to: 1) inadequate screening and referral for testing [21];2) test results not reaching care providers and patients on time; 3 ) inefficient processes to recall patients and pre-treatment losses to follow-up; and 3) the need for referral and/or confirmatory testing for treatment initiation in some settings. Many countries have also struggled to provide MDR-TB treatment services to keep pace with the roll-out of improved diagnostics, at least partly due to the high cost of second-line drugs.

In most countries, data are not routinely collected to monitor the proportion of diagnosed patients who start treatment or the time from diagnosis to treatment initiation (the "treatment gap"). The available data suggest that treatment initiation following diagnosis is one of the weakest links in the cascade of care. In South Africa this treatment gap is as large as $32-54 \%$ in drug-resistant TB patients [22].

\section{Impact measurement: does Xpert MTB/RIF improve patient outcomes?}

A minimal set of Xpert-specific impact data was available at the time of the WHO endorsement (e.g. time to diagnosis) and data reviewed by WHO included a cost-effectiveness analysis and an affordability study $[23,24]$.

Since 2011, a number of randomised controlled trials have been conducted to measure important patient outcomes relevant to the roll-out of Xpert MTB/RIF [25-28]. A number of recent studies conducted under routine programmatic conditions have shown that upfront drug susceptibility testing with Xpert has dramatically improved access to MDR-TB diagnosis, with the number of MDR cases detected in adults and children increasing by up to eight-fold, with a two-fold increase in the rate of bacteriologically confirmed TB [26, 29-32]. Other studies have shown minimal early impact in mortality, and have been interpreted as establishing an absence of impact on patient outcomes. However, mortality results of published studies were largely inconclusive due to studies being underpowered to detect important effects on mortality. Furthermore, high levels of empiric treatment among smear-negative TB suspects [31] may have further reduced the effects of new diagnostics on outcomes. The benefits from reduced overtreatment are hard to capture, and change in provider behaviour may be slow, an issue known from the use of more sensitive rapid diagnostic tests in the diagnosis of malaria [33]. Perhaps most importantly, Xpert MTB/RIF roll-out has not clearly defined the linkage of newly diagnosed patients to rapid treatment initiation and follow-up, and this critical element has been largely at the clinician's discretion. Thus, gaps at all stages of the TB care cascade have undermined the impact of the Xpert technology.

Diagnosis of patients, particularly MDR-TB patients, has undeniably improved. However, more data are needed to answer critical questions with respect to individual patient impact, public health impact (including a broader transmission perspective), and the potential cost savings to the health system. For novel diagnostic tools, countries need detailed guidance and decision-making tools, informed by programmatic data, to design implementation strategies specific to their settings $[34,35]$. Furthermore, the roll-out of novel tools should be accompanied from the start by impact measurement within routine data collection and guidance to providers on decision-making based on information from a test. 


\section{Private sector access}

Roll-out of new diagnostic technologies, including Xpert, has largely focused on the public sector. However, the private sector plays a major role in TB care and treatment. For example, in India and Pakistan $70-80 \%$ of first contact with health services occurs in the private sector [36]. SALJE et al. [37] demonstrated that implementation of Xpert MTB/RIF in the public sector in India would have limited impact on TB incidence. To ensure optimal impact it is necessary to engage the whole private healthcare sector and provide adequate referral systems, improved treatment quality and increased resources across all healthcare sectors [37].

However, the private sector is not yet eligible for concessional Xpert MTB/RIF pricing. The manufacturer charges higher reagent and instrument prices for the private sector, and there are significant additional costs, including import duties and margins imposed by distributors, intermediaries and laboratories. PuRI et al. [38] surveyed 12 highly privatised HBDCs and found no commercial availability of Xpert in the private sector in six of the 12 countries. In the remaining six countries, the average price charged by private laboratories was USD $\$ 68.73$, with a range of US $\$ 30.26$ to US $\$ 155.44$. Thus, use of Xpert appears to be price constrained and limited in the private sector, which undoubtedly translates into low levels of access and blunted impact. There have been innovative attempts to improve access in the private sector. For instance, engagement of private providers in Pakistan using a social franchising model has demonstrated significant impact on patient care [39, 40]. The Initiative for Promoting Affordable and Quality TB diagnostic tests in India has made Xpert nearly 50\% more affordable than the prevailing market price, with substantial increases in testing volumes in the private sector [38]. These strategies may serve as a model for expansion within India and Pakistan, as well as other countries.

\section{Lessons learnt to inform new test developers}

The scale-up of Xpert has inspired diagnostic companies to invest in TB diagnostic development, and the current TB diagnostics pipeline includes more than 50 companies developing new TB diagnostics, more than 30 of which are molecular. Xpert roll-out has enhanced our understanding of target product profiles. Considerations around clinical impact and operational aspects should be integral components of these target product profiles [41]. It is also important for test developers to understand how novel diagnostic tests will fit into multi-test algorithms for TB care.

The importance of integrating advances in information technology into the design of diagnostic platforms cannot be overemphasised. There is increasing coordination of efforts to improve connectivity solutions in many aspects of healthcare delivery, including prevention, diagnosis, data collection, treatment and adherence monitoring, surveillance and supply chain management [42]. Product developers should integrate connectivity hardware and software into their designs at an early stage in product development.

\section{Lessons learnt to inform roll-out of future technologies}

Several key lessons learnt from the roll-out of Xpert MTB/RIF may inform more efficient and effective scale-up of other technologies, both in TB and other diseases (table 1).

\section{Table 1 Lessons learnt to inform roll-outs of new technologies}

A complete package of solutions including policy/regulatory, training, quality assurance, supply chain management and connectivity needs to be available at product launch

Health system strengthening should be up-scaled in alignment with any expansion of diagnostic capacity, with a focus on improving linkages to care and routine impact measurement

Planning and budgeting for diagnostics implementation, whether donor or government funded, should include a comprehensive implementation approach including systems strengthening

Advocacy and information sharing among test operators, clinicians and the patient community is needed to raise awareness of and demand for testing

Expanded engagement and concessionary pricing for private sector providers is needed to expand the impact

Diagnostics connectivity is a pre-requisite for future technologies; successful implementation of connectivity solutions will require processes of change at many levels, global commitment, a roadmap and pro-active champions

Strong coordination, both globally among donors and partners, and within countries, between National Tuberculosis Programmes, National AIDS Control Programmes and other stakeholders, is essential for efficient rollout

Reliance on donor funding to support scale-up of new diagnostics should be reduced by advocating for inclusion of costs in national government budgets 
Market priming and demand creation are also essential to ensure tests are used in the most cost-effective way. Clinicians need to be sensitised on new diagnostic algorithms in order to drive appropriate use and increase test uptake. Similarly, public health officials should work with patients and their communities to gain their support in advocating for access to new technologies.

Rather than focusing on implementing a new technology, we advocate the need to consider a holistic packaged solution approach. All components of the diagnostic solution must be available at the time of product launch and effectively disseminated to countries. Countries must be actively engaged and supported to translate global policy into an actionable implementation plan, with a clear framework for impact measurement. Critically, a systems strengthening approach is essential for any new technologies, including true point-of-care platforms to ensure patient impact, and an expansion of treatment capacity to meet the needs of an increase in diagnosed patients, and must be tackled in parallel. Models for providing the appropriate level of support to countries in scaling-up new technologies are being refined as a result of Xpert MTB/RIF implementation, to ensure that support provided results in genuine local capacity strengthening and sustainable benefits.

\section{Conclusion}

New diagnostic technologies will play a critical role in improving TB control and the global targets envisioned by the END TB Strategy [43]. Indeed, Xpert MTB/RIF has paved the way for meeting a key component of the first pillar of the END TB Strategy; early diagnosis of TB including universal drug susceptibility testing. However, diagnostic tests alone, if not implemented with a comprehensive package of accompanying tools and within the context of a strengthened health system, may fail to demonstrate the expected benefit.

A strong focus on improving the entire TB care cascade, engagement of all health service providers (public and private), and building country capacity for using local data for decision making are all required to ensure an efficient and impactful introduction of next generation technologies. Connectivity solutions will be integral to success. Adequate and early engagement of country leadership and preparation of systems for the introduction of new tools are paramount. Finally, strong coordination of various stakeholders, advocacy from patients and civil society, and political leadership both at the national and global level will be essential.

\section{References}

1 Boehme C, Nabeta P, Hillemann D, et al. Rapid molecular detection of tuberculosis and rifampin resistance. N Engl J Med 2010; 363: 1005-1015.

2 Boehme CC, Nicol MP, Nabeta P, et al. Feasibility, diagnostic accuracy, and effectiveness of decentralised use of the Xpert MTB/RIF test for diagnosis of tuberculosis and multidrug resistance: a multicentre implementation study. Lancet 2011; 377: 1495-1505.

3 Weyer K, Mirzayev F, Migliori GB, et al. Rapid molecular TB diagnosis: evidence, policy making and global implementation of Xpert MTB/RIF. Eur Respir J 2013; 42: 252-271.

4 World Health Organization. Policy statement: automated real-time nucleic acid amplification technology for rapid and simultaneous detection of tuberculosis and rifampicin resistance: Xpert MTB/RIF. Policy statement. Geneva, World Health Organization, 2011.

5 Ramsay ARC, Steingart KR, Cunningham J, et al. Translating tuberculosis research into global policies: the example of an international collaboration on diagnostics. Int J Tuberc Lung Dis 2011; 15: 1283-1293.

6 World Health Organization. Automated real-time nucleic acid amplification technology for rapid and simultaneous detection of tuberculosis and rifampicin resistance: Xpert MTB/RIF assay for the diagnosis of pulmonary and extrapulmonary TB in adults and children. Policy update. Geneva, World Health Organization, 2013.

7 World Health Organization. Updated: Xpert MTB/RIF implementation manual technical and operational "how-to": practical considerations. Geneva, World Health Organization, 2014.

8 Qin ZZ, Pai M, Van Gemert W, et al. How is Xpert MTB/RIF being implemented in 22 high tuberculosis burden countries? Eur Respir J 2014; 45: 549-554.

9 Medecins Sans Frontieres, Stop TB Partnership. Out of Step 2015 TB Policies in 24 Countries. Geneva, Medecins Sans Frontieres, 2015.

10 Scott L, Albert H, Gilpin C, et al. Multicenter feasibility study to assess external quality assessment panels for Xpert MTB/RIF assay in South Africa. J Clin Microbiol 2014; 52: 2493-2499.

11 Kik SV, Denkinger CM, Chedore P, et al. Replacing smear microscopy for the diagnosis of tuberculosis: what is the market potential? Eur Respir J 2014; 43: 1793-1796.

12 Raizada N, Sachdeva KS, Sreenivas A, et al. Feasibility of decentralised deployment of Xpert MTB/RIF test at lower level of health system in India. PLoS One 2014; 9: e89301.

13 Creswell J, Codlin AJ, Andre E, et al. Results from early programmatic implementation of Xpert MTB/RIF testing in nine countries. BMC Infect Dis 2014; 14: 2.

14 Molapo S, Berrie L, Marokane P, et al. GeneXpert module failures: South Africa's Xpert MTB/RIF national programme experience and impact on costs. Int J Tuberc Lung Dis 2014; 18: Suppl. 1, S1.

15 Mookherji S, Mehl G, Kaonga N, et al. Unmet need: improving mHealth evaluation rigor to build the evidence base. J Health Commun 2015; 20: 1224-1229.

16 World Health Organization, European Respiratory Society. The role of e/mHealth in tuberculosis and tobacco control. 2015. Available from: www.who.int/tb/features_archive/emHealthinTBandtobaccocontrol.pdf

17 Denkinger CM, Dolinger D, Schito M, et al. Target product profile of a molecular drug-susceptibility test for use in microscopy centers. J Infect Dis 2015; 211: Suppl 2, S39-S49. 
18 Andre E, Isaacs C, Affolabi $\mathrm{D}$, et al. Connectivity of diagnostic technologies: improving surveillance and accelerating TB elimination. Int J Tuberc Lung Dis 2016. In Press.

19 World Health Organization. Joint Inter-Ministerial Policy Dialogue on eHealth Standardization and Second WHO Forum on eHealth Standardization and Interoperability. Geneva, World Health Organization, 2014.

20 Starks AM, Avilés E, Cirillo DM, et al. Collaborative effort for a centralized worldwide tuberculosis relational sequencing data platform. Clin Infect Dis 2015; 61: Suppl 3, S141-S146.

21 Das J, Kwan A, Daniels B, et al. Use of standardised patients to assess quality of tuberculosis care: a pilot, cross-sectional study. Lancet Infect Dis 2015; 15: 1305-1313.

22 Low M, Thom A. HIV positive: is South Africa winning? NSP Review 2013; 9: 1-45.

23 Pantoja A, Fitzpatrick C, Vassall A, et al. Xpert MTB/RIF for diagnosis of tuberculosis and drug-resistant tuberculosis: a cost and affordability analysis. Eur Respir J 2013; 42: 708-720.

24 Vassall A, van Kampen S, Sohn H, et al. Rapid diagnosis of tuberculosis with the Xpert MTB/RIF assay in high burden countries: a cost-effectiveness analysis. PLoS Med 2011; 8: e1001120.

25 Theron G, Zijenah L, Chanda D, et al. Feasibility, accuracy, and clinical effect of point-of-care Xpert MTB/RIF testing for tuberculosis in primary-care settings in Africa: a multicentre, randomised, controlled trial. Lancet 2014; 383: 424-435.

26 Durovni B, Saraceni V, van den Hof S, et al. Impact of replacing smear microscopy with Xpert MTB/RIF for diagnosing tuberculosis in Brazil: a stepped-wedge cluster-randomized trial. PLoS Med 2014; 11: e1001766.

27 Churchyard GJ, Stevens WS, Mametja LD, et al. Xpert MTB/RIF versus sputum microscopy as the initial diagnostic test for tuberculosis: a cluster-randomised trial embedded in South African roll-out of Xpert MTB/RIF Lancet Glob Health 2015; 3: e450-e457.

28 Cox HS, Mbhele S, Mohess N, et al. Impact of Xpert MTB/RIF for TB diagnosis in a primary care clinic with high TB and HIV prevalence in South Africa: a pragmatic randomised trial. PLoS Med 2014; 11: e1001760.

29 Raizada N, Sachdeva KS, Sreenivas A, et al. Catching the missing million: experiences in enhancing TB and DR-TB detection by providing upfront Xpert MTB/RIF testing for people living with HIV in India. PLoS One 2015; 10: e0116721.

30 Raizada N, Sachdeva KS, Nair SA, et al. Enhancing TB case detection: experience in offering upfront Xpert MTB/RIF testing to pediatric presumptive TB and DR TB cases for early rapid diagnosis of drug sensitive and drug resistant TB. PLoS One 2014; 9: e105346.

31 Sachdeva KS, Raizada N, Sreenivas A, et al. Use of Xpert MTB/RIF in decentralized public health settings and its effect on pulmonary TB and DR-TB case finding in India. PLoS One 2015; 10: e0126065.

32 Trajman A, Durovni B, Saraceni V, et al. Impact on patients' treatment outcomes of XpertMTB/RIF implementation for the diagnosis of tuberculosis: follow-up of a stepped-wedge randomized clinical trial. PLoS One 2015; 10: $\mathrm{e} 0123252$.

33 Masanja IM, Selemani M, Amuri B, et al. Increased use of malaria rapid diagnostic tests improves targeting of anti-malarial treatment in rural Tanzania: implications for nationwide rollout of malaria rapid diagnostic tests. Malar J 2012; 11: 221.

34 Pho MT, Deo S, Palamountain KM, et al. Optimizing tuberculosis case detection through a novel diagnostic device placement model: the case of Uganda. PLoS One 2015; 10: e0122574.

35 Langley I, Lin H-H, Egwaga S, et al. Assessment of the patient, health system, and population effects of Xpert MTB/RIF and alternative diagnostics for tuberculosis in Tanzania: an integrated modelling approach. Lancet Glob Health 2014; 2: e581-e591.

36 Baloch NA, Pai M. Tuberculosis control: business models for the private sector. Lancet Infect Dis 2012; 12: 579-580.

37 Salje H, Andrews JR, Deo S, et al. The importance of implementation strategy in scaling up Xpert MTB/RIF for diagnosis of tuberculosis in the Indian health-care system: a transmission model. PLoS Med 2014; 11: e1001674.

38 Puri L, Oghor C, Denkinger CM, et al. Xpert MTB/RIF for tuberculosis: access and price in highly privatized health markets. Lancet Glob Health 2016; 4: e94-e95

39 Khan AJ, Khowaja S, Khan FS, et al. Engaging the private sector to increase tuberculosis case detection: an impact evaluation study. Lancet Infect Dis 2012; 12: 608-616.

40 Dowdy DW, Lotia I, Azman AS, et al. Population-level impact of active tuberculosis case finding in an Asian megacity. PLoS One 2013; 8: e77517.

41 Denkinger CM, Kik SV, Cirillo DM, et al. Defining the needs for next generation assays for tuberculosis. J Infect Dis 2015; 211: Suppl 2, S29-S38.

42 Denkinger CM, Grenier J, Stratis AK, et al. Mobile health to improve tuberculosis care and control: a call worth making. Int J Tuberc Lung Dis 2013; 17: 719-727.

43 World Health Organization. The End TB Strategy. Geneva, World Health Organization, 2015. 